\title{
La actividad física y el deporte saludable en exdeportistas de élite
}

\section{Former elite athletes' current sport and physical activity}

\section{Atividade física e esporte saudável em ex-atletas de elite}

\author{
Torregrossa, M. ${ }^{1}$; Conde, E. ${ }^{2}$; Perez-Rivases, A. ${ }^{1}$; Soriano, G. ${ }^{1}$ y Ramis, Y. ${ }^{1}$ \\ ${ }^{1}$ Universitat Autònoma de Barcelona, España; ${ }^{2}$ Universidad Católica San Antonio Murcia, España
}

\begin{abstract}
RESUMEN
El propósito de este trabajo es doble: (a) describir los aspectos motivacionales y beneficios que obtienen los exdeportistas de élite que se mantienen físicamente activos y las barreras que perciben los físicamente inactivos, y (b) probar un modelo motivacional que pueda predecir la práctica de actividad física en esta población. Se realizó un estudio transversal. Participaron 317 exdeportistas de élite (Medad $=36.5, \mathrm{DS}=7.18$ años). Completaron un cuestionario en línea de motivaciones, cantidad de ejercicio, estados de cambio, beneficios y barreras. Los beneficios principales fueron la diversión, la revitalización y la buena salud. Las barreras principales para los inactivos (12.9\%) fueron los conflictos con otras actividades, su forma física actual y el hecho de haber perdido acceso a recursos e instalaciones. Para promover actividad física saludable en la población de exdeportistas inactivos los programas de promoción deberían enfatizar la buena salud, el reconocimiento social y la competición, que predicen positivamente la regulación intrínseca y la actividad física.
\end{abstract}

Palabras clave: Exdeportistas de elite, Motivación, Deporte y Actividad Física, Transiciones de Carrera, Retirada Deportiva.

\begin{abstract}
The purpose of this study is twofold: (a) to describe the motivational aspects and gains obtained by physically active former elite athletes and the barriers experienced by inactive former elite athletes comparing their reported exercise amount, and (b) to test whether the different motivations would predict their reported exercise amount. Using a crosssectional survey design, 317 former athletes (Mage $=36.5, \mathrm{SD}=7.18$ years) completed an online questionnaire assessing motivation, self-reported exercise and sport practice, stages of change, gains, and barriers. The main gains reported by former athletes were enjoyment, revitalization and positive health. Physically inactive former athletes $(12.9 \%)$ spotted as the main barriers conflict with other activities, current physical fitness and lost access to facilities and resources. To promote healthy physical activity in the physically inactive population of former elite athletes, policy makers and physical activity organizers should emphasize positive health, social recognition and competition motives, which positively predict intrinsic regulation and vigorous physical activity.
\end{abstract}

Keywords: Former Elite Athletes, Motivation, Sport and Physical Activity, Career transitions, Sport retirement. 


\section{Deporte después del deporte}

\section{RESUMO}

O objetivo deste artigo é duplo: (a) descrever os aspectos motivacionais e benefícios obtidos por exdeportistas elite é fisicamente ativo e barreiras percebidas por fisicamente inativos, e (b) a testar um modelo motivacional que pode prever prática de atividade física nesta população. Um estudo transversal foi realizado. Havia 317 ex-atletas de elite (Medad = 36,5, DS = 7,18 anos). Eles preencheram um questionário on-line de motivações, quantidade de exercício, estados de mudança, benefícios e barreiras. Os principais benefícios foram diversão, revitalização e boa saúde. As principais barreiras para os inativos $(12,9 \%)$ foram os conflitos com outras atividades, sua forma física atual e o fato de ter perdido acesso a recursos e instalações. Para promover a atividade física saudável na população de ex-atletas inativos, os programas de promoção devem enfatizar a boa saúde, o reconhecimento social e a competição, que preveem positivamente a regulação intrínseca e a atividade física.

Palavras chave: Desportistas de elite, Motivação, Esporte e atividade física, Transições de carreira, Retiro esportivo

\section{INTRODUCCIÓN}

El proceso de retirada del deporte ha sido ampliamente estudiado en la literatura científica sobre transiciones de la carrera deportiva (ver Jordana, Torregrossa, Ramis y Latinjak, 2017; Park, Lavallee, y Tod, 2013 para revisión). La mayor parte de esta investigación se ha focalizado en los aspectos sociales y vocacionales de la retirada (e.g., problemas de adaptación, dificultades para establecer una carrera profesional alternativa) mientras que otros aspectos más relacionados con la salud física como la adopción de un estilo de vida activo o los cambios corporales después de la retirada han recibido menos atención (Abel y Carreiro, 2011; Stephan, Torregrosa, y Sanchez, 2007). En este estudio, exploramos aspectos motivacionales implicados en los niveles actuales de práctica de deporte y actividad física de exdeportistas de élite retirados. Los beneficios percibidos por aquellos que practican deporte y actividad física, así como las barreras percibidas por aquellos que no lo hacen. Paradójicamente, tal y como sugiere la literatura científica, el hecho de haber sido deportista de élite en lugar de ser un factor protector, representa un factor de riesgo para la salud una vez se da la retirada de la práctica deportiva. De acuerdo con Witkowski y Spangenburg (2008) “... después de la retirada, si ellos [los deportistas de élite] adoptan un estilo de vida que no incluye una cantidad significativa de actividad física, el riesgo de enfermedad crónica se incrementa de manera dramática" (p.953). En consecuencia, el interés en promover tanto el deporte recreativo como la actividad física saludables entre los deportistas de élite retirados es especialmente relevante.

Deporte y actividad física en deportistas de élite retirados
La retirada deportiva ha sido la primera y probablemente la más estudiada de las transiciones de la carrera deportiva (Mihovilovic, 1968; Jordana et al., 2017; Park et al., 2013). Después de más de tres décadas estudiando la retirada del deporte la mayoría de los esfuerzos en el ámbito de la psicología se han dedicado sobre todo a los problemas relacionados con la transición y los procesos de adaptación (Dimoula, Torregrosa, Psychountaki, y Gonzalez, 2013; Navel y de Tychey, 2011; Stambulova, Alfermann, Statler, y Côté, 2009) y más recientemente a la promoción de carreras duales (i.e., combinar el deporte con los estudios y/o un trabajo) como una aproximación preventiva para optimizar el proceso de transición y minimizar los problemas asociados a la retirada (López de Subijana, Barriopedro y Conde, 2015; Stambulova y Wylleman, 2015; Torregrosa, Ramis, Pallarés, Azócar, y Selva, 2015). Sin embargo, la adopción de un estilo de vida saludable y la calidad de vida de los deportistas cuando se retiran del deporte de élite no se ha estudiado en profundidad.

Los datos empíricos sobre deportistas de élite retirados y sus niveles de práctica deportiva y actividad física saludable son escasos. En una muestra de adultos finlandeses, Bäckmand, Kujala, Sarna, y Kaprio, (2010) reportaron que el 54\% pertenecían al grupo más activo en términos de actividad física versus el $44 \%$ de sus coetáneos no deportistas. A pesar de que los exdeportistas son más activos como grupo que las personas no deportistas, los datos presentaban un $46 \%$ de deportistas de élite retirados que aparentemente, no practicaban suficiente deporte y actividad física saludable.

Otra fuente de datos empíricos sobre la actividad física practicada por deportistas retirados es 


\section{Torregrossa, M.; Conde, E.; Perez-Rivases, A.; Soriano, G. 1 y Ramis, Y.}

la de los exdeportistas universitarios en los EEUU. Reifsteck y Gill (2011) encontraron una relación positiva entre la identidad deportiva y los niveles de actividad física. Los mismos autores encontraron más tarde que el papel predictivo de la identidad deportiva para la actividad física de exdeportistas es más fuerte cuando esta se combina con una actitud positiva hacia el ejercicio (Reifsteck, Gill, y Labban, 2016). Basándose también en la experiencia de exdeportistas universitarios, Abel y Carreiro (2011) concluyeron que el hecho de haber perdido el acceso a las instalaciones, la obligación de realizar entrenamientos y rutinas sin supervisión externa conjuntamente con la motivación extrínseca, son algunas de las barreras principales percibidas por los exdeportistas para la práctica de actividad física saludable y/o deporte recreativo. Estos autores también sugieren que hay una necesidad de reconceptualizar lo que muchos deportistas entienden como actividad física: 'muchos deportistas no entienden que las actividades diarias típicas como pasear al perro o ir en bicicleta al trabajo cuentan como actividad física (p.59)'. Los escasos datos sobre las motivaciones para la actividad física saludable y el deporte recreativo por parte de deportistas de élite retirados y las diferencias entre estos y los deportistas universitarios retirados (en términos de edad, carrera deportiva, etc.) justifican la contribución del presente estudio.

\section{Motivación para la práctica de actividad física saludable y deporte recreativo}

Los comportamientos sedentarios y la falta de actividad física en adultos sanos para mantener o mejorar su salud es una preocupación de la sociedad occidental actual. El American College of Sports Sciences and Medicine recomienda que "para promover y mantener la salud, todos los adultos sanos entre 18 y 65 años necesitan realizar actividad física de intensidad moderada (de resistencia) un mínimo de 30 minutos cinco días por semana, o actividad física vigorosa un mínimo de 20 minutos tres días por semana' (p.1423; Haskell et al., 2007). Sin embargo, en las sociedades modernas, solo una minoría de adultos sanos reportan estar en niveles de ejercicio físico en un nivel compatible con la mayoría de directrices de salud pública (Teixeira, Carraça, Markland, Silva, y Ryan, 2012). La presencia o ausencia de motivación es un factor crítico para apoyar la realización de ejercicio habitual, es por ello que entender el papel de la motivación como determinante de la participación en el ejercicio físico se ha estudiado en un amplio rango de contextos de actividad física y con múltiples poblaciones incluyendo: adultos sanos (e.g., Scarapicchia, Amireault, Faulkner, y Sabiston, 2016), adultos jóvenes/estudiantes universitarios (e.g, Moreno-Murcia, Huéscar, y Cervelló, 2012; Samperio, Jiménez-Castuera, Lobato, Leyton y Claver, 2016), adultos mayores (Marcos Pardo, Orquín Castrillón, Belando Pedreño, y MorenoMurcia, 2014), y poblaciones clínicas (e.g., Carek, Laibstain, y Carek, 2011). Tal y como resumen Teixeira et al. (2012) los resultados de estas diversas poblaciones muestran una relación positiva consistente entre formas más autónomas de motivación y la realización de actividad física saludable, sin embargo, ninguno de estos trabajos se ha centrado aún en los exdeportistas de élite.

Basándose en la teoría de la motivación autodeterminada (SDT por sus siglas en inglés; Deci y Ryan, 2000), Ingledew, Markland, y Ferguson (2009) propusieron tres niveles distintos de motivación hacia el ejercicio (i.e., disposicionales, participativos y regulatorios). Los motivos disposicionales se refieren al contenido de los objetivos de los individuos para la vida en general (e.g., valores morales, objetivos vitales), los motivos participativos se refieren al contenido de los objetivos de los individuos para un ámbito conductual particular (e.g., diversión, reconocimiento social, buena salud), y los motivos regulatorios, o regulación conductual, se refieren al locus de causalidad percibida de los objetivos conductuales de los individuos (e.g., intrínsecos, extrínsecos). En población adulta, estos autores encontraron que las regulaciones conductuales son predichas por los motivos participativos, específicamente los motivos de afiliación y desafío predicen la motivación intrínseca, y el reconocimiento social y los motivos de peso/apariencia predicen la motivación extrínseca. Posteriormente los mismos autores establecen una distinción entre motivos (i.e., lo que las personas quieren del ejercicio) y beneficios (i.e., lo que las personas realmente obtienen del ejercicio; Ingledew, Markland, y Strömmer, 2014). En dicho estudio se obtuvieron dos resultados relevantes, en primer lugar, la regulación conductual autónoma tuvo efectos positivos en la cantidad de ejercicio y, en segundo lugar, se comprobó que los beneficios percibidos eran moderadores de los efectos de los motivos en la regulación conductual (e.g., el motivo de buena salud predecía la regulación autónoma

Cuadernos de Psicología del Deporte, 19, 2 (mayo) 


\section{Deporte después del deporte}

cuando el beneficio de salud percibido era alto). La investigación previa ha mostrado que los motivos participativos de apariencia también son importantes para empezar a hacer ejercicio, sin embargo, para que esta participación sea sostenida son más importantes otros motivos como la afiliación, el desafío o la mejora de la salud (Ingledew, Markland, y Medley, 1998).

A pesar de que los beneficios de la actividad física son bien conocidos, una parte significativa de la población es físicamente inactiva (Teixeira et al., 2012). Varios estudios han explorado las barreras percibidas para la realización de actividad física desde finales de los años noventa del siglo XX hasta la actualidad. Zunft et al., (1999) con una muestra representativa de adultos a nivel europeo, encontraron que las barreras percibidas más importantes para incrementar la actividad física eran las obligaciones de trabajo o de estudios a la vez que las creencias de los participantes de no reconocerse como 'una persona deportiva'. King et al., (2000) con una muestra de mujeres de mediana edad y mayores encontraron que las obligaciones diarias y la falta de energía estaban entre las barreras evaluadas como más importantes para hacer ejercicio. Reichert, Barros, Domingues y Hallal, (2007) con una muestra de adultos brasileños encontraron que la falta de dinero y el sentirse demasiado cansados eran las barreras más reportadas. En resumen, las barreras percibidas se pueden dividir en: (a) conflicto con otras actividades, y (b) falta de recursos sea personales o estructurales. Debido a la falta de datos empíricos sobre las barreras percibidas para la actividad física y el deporte recreativo en exdeportistas de élite este estudio tiene también como objetivo describir las barreras percibidas en esta población.

Cuando estudiamos motivos, beneficios y barreras para ser físicamente activo, debemos definir claramente cuando consideramos que un individuo es activo o inactivo. De acuerdo con el modelo teórico de Prochaska y DiClemente (1984), ser activo o inactivo no es una cuestión de todo o nada. El modelo describe las distintas fases por las que las personas pasan para la adquisición y mantenimiento de un comportamiento (i.e., precontemplación, contemplación, preparación, acción y mantenimiento). Este modelo se ha utilizado para explicar la implicación de distintas poblaciones en la realización de actividad física (De Bourdeaudhuij y Sallis, 2002; Sørensen y Gill, 2008). En referencia a la relación entre estados de cambio y motivos Ingledew et al. (1998) encontraron que los motivos extrínsecos (e.g., imagen física) dominan durante las primeras etapas de la iniciación en actividad física mientras que los motivos intrínsecos (diversión) son importantes para la progresión y el mantenimiento de la actividad. Por ello, en nuestro estudio ubicamos a los exdeportistas de élite en las distintas etapas y comparamos su motivación en función de dicha variable.

En la actualidad, son escasos los estudios que han proporcionado datos sobre aspectos motivacionales relacionados con la actividad física saludable y el deporte recreativo practicado por exdeportistas de élite. Así, el primer objetivo de este trabajo es describir los aspectos motivacionales, los beneficios obtenidos por exdeportistas de élite activos físicamente, las barreras experimentadas por los físicamente inactivos y la cantidad de ejercicio físico reportado. El segundo objetivo es comprobar si las relaciones entre los distintos tipos de motivos y regulaciones podrían determinar la cantidad de ejercicio físico reportado, con la intención de detectar que motivos deberían ser enfatizados para promover la actividad física y el deporte recreativo para exdeportistas inactivos. Nuestras hipótesis son: (a) los exdeportistas activos mostrarán un perfil motivacional más saludable y reportarán más actividad física que los deportistas inactivos, (b) los motivos intrínsecos y la regulación intrínseca predecirán significativamente la actividad física y el deporte recreativo.

\section{MATERIAL Y MÉTODOS}

\section{Participantes}

La muestra incluye 317 exdeportistas de élite $\left(M_{\text {edad }}=36.5\right.$, $D S=7.18$, Rango $=22-58)$ retirados desde un promedio de 6.43 años $(D S=3.57)$ antes de la recogida de datos. Ciento sesenta y tres $(51.4 \%)$ españoles, la mayoría provenientes de las bases de datos de un centro de alto rendimiento deportivo $(89.6 \%)$ y una minoría de otros deportes profesionales $(10.4 \%)$. Ciento cincuenta y cuatro $(48.6 \%)$ colombianos, reclutados a través del comité Olímpico Colombiano. Los deportistas eran 135 mujeres (42.6\%) y 182 hombres $(57.4 \%)$ y habían competido en deportes Olímpicos (79.5\%), Paralímpicos $(2.8 \%)$ y deportes no incluidos en el programa Olímpico (17.7\%). De los 317 exdeportistas que empezaron a contestar la batería de cuestionarios 297 (93.7\%) la completaron. Hemos mantenido todas las medidas y se reporta la ratio exacta de 


\section{Torregrossa, M.; Conde, E.; Perez-Rivases, A.; Soriano, G. 1 y Ramis, Y.}

respuesta para cada cuestionario en la sección de procedimiento.

\section{Instrumentos}

Motivos para el ejercicio. Los motivos de participación se midieron con la sección de motivos de la versión en español del Inventario de Motivos y Beneficios para el Ejercicio (Exercise Motives and Gains Inventory, EMGI-2; Ingledew et al., 2014; Markland y Ingledew, 1997). Dicho instrumento ha sido traducido y publicado en un manual de actividad física (Capdevila, 1999) y no consta su validación y publicación en una revista de publicación por pares, es por ello que en el apartado de resultados se reportan las alfas de Cronbach de cada subescala y se comparan con las de la versión original en inglés. Un total de 51 ítems agrupados en 14 escalas (i.e., Buena salud, Diversión, Revitalización, Evitación de mala salud, Gestión del estrés, Agilidad, Fuerza y resistencia, Control del peso, Apariencia, Competición, Afiliación, Desafío, Presiones de salud, y Reconocimiento social). Todos los ítems se inician con la raíz "Personalmente, hago ejercicio o practico deporte a nivel recreativo (o debería hacer ejercicio practica deporte recreativo) ..." y un ejemplo de ítem es “...para pasar tiempo con amigos”. Las opciones de respuesta presentaban un rango de 0 (nada cierto para mi) a 4 (muy cierto para mi).

Regulación conductual. La regulación conductual se midió utilizando la versión Española del Cuestionario de Regulación Conductual del Ejercicio versión 3 (Behavioral Regulation in Exercise Questionnaire; González-Cutre, Sicilia, y Fernández, 2010; Wilson, Rodgers, Loitz, y Scime, 2006) revisada por los autores del BREQ-2 original (Markland y Tobin, 2004). Un total de 24 ítems agrupados en 6 escalas (i.e., Regulación intrínseca, Regulación integrada, Regulación identificada, Regulación introyectada, Regulacion externa y Amotivación. Todos los ítems responden a la pregunta “¿Por qué te implicas o deberías implicarte en hacer ejercicio físico $\mathrm{y} / \mathrm{o}$ deporte recreativo?" y un ejemplo de ítem fue “... no veo la razón para hacer ejercicio físico o deporte recreativo". El rango de respuestas iba desde 0 (nada cierto en mi caso) hasta 4 (muy cierto en mi caso).

Cantidad de ejercicio físico autoinformada. Siguiendo (Ingledew et al., 2014) utilizamos una versión española para preguntar a los participantes “¿Durante los últimos 7 días, cuantas veces has hecho el siguiente tipo de ejercicio durante al menos 30 minutos?" Los 3 tipos de ejercicio se correspondían con (a) vigoroso (e.g., levantar pesos, cavar, hacer aerobic o similar, montar en bicicleta a marcha rápida o ritmo intenso), (b) moderado (e.g., transportar pesos ligeros, montar en bicicleta a ritmo regular, jugar un partido de dobles de tenis), y (c) ligero (e.g., caminar para ir y volver del trabajo como mínimo 10 minutos, caminar por ocio).

Estados de cambio. Seguimos el planteamiento de Sørensen y Gill (2008) para clasificar a los deportistas respecto a los estados de cambio en relación con la actividad física y el deporte recreativo. El ítem se formuló del siguiente modo "A continuación verás algunas afirmaciones que describen varios niveles de actividad física. Por favor, indica el nivel que mejor describe tu situación actual". Las alternativas de respuesta fueron: (a) en la actualidad no soy físicamente activo y no tengo planes de estarlo en los próximos 6 meses (precontemplación), (b) en la actualidad no soy físicamente activo pero tengo planes de estarlo en los próximos 6 meses (contemplación), (c) en la actualidad soy algo activo físicamente, pero no con regularidad (preparación), (d) en la actualidad soy físicamente activo, pero lo soy desde hace menos de 6 meses (acción), y (e) en la actualidad soy físicamente activo, y lo he sido durante más de 6 meses (mantenimiento).

Beneficios del ejercicio. Siguiendo a Ingledew et al., (2014) se generó un ítem de beneficio correspondiente a cada ítem de motivos dando como resultado la sección de beneficios del EMGI-2 de 51 ítems agrupados en 14 subescalas. La raíz fue "Mi experiencia personal con el ejercicio físico saludable y el deporte recreativo ha sido que ..." y un ejemplo de ítem fue "...me ha permitido pasar tiempo con amigos". El rango de las opciones de respuesta fue desde 0 (nada cierto para mi) a 4 (muy cierto para mi).

Barreras para el ejercicio. Desarrollamos un cuestionario ad hoc para medir específicamente las Barreras para el Ejercicio físico y el Deporte recreativo percibidas por Exdeportistas de élite (BEDE). Este cuestionario se inspiró en el Autoinforme de barreras percibidas para el ejercicio físico (ABPEF) de Niñerola, Capdevila, y Pintanel (2006). Se realizaron dos reuniones de expertos para generar la nueva escala. En la primera se discutieron los ítems originales del ABPEF para evaluar si se

Cuadernos de Psicología del Deporte, 19, 2 (mayo) 


\section{Deporte después del deporte}

adaptaban a la población de exdeportistas de élite. Después de concluir que se debían incluir ítems orientados a evaluar las barreras específicas percibidas por los exdeportistas de élite, se generó un pool de 68 ítems evaluando seis aspectos: (a) Imagen corporal actual, (b) Condición física pobre, (c) Compatibilización, (d) Problemas físicos derivados de la carrera deportiva, (e) Problemas psicológicos derivados de la carrera deportiva (i.e., burnout), y (f) Acceso a recursos e instalaciones. En la segunda reunión de expertos, se les pidió que priorizasen los ítems que evaluaban mejor esos seis aspectos y se propusieron 4 ítems para cada subescala. Se pidió a tres revisores independientes (i.e., exdeportistas de élite con un doctorado en psicología del deporte) que no habían participado en las reuniones de expertos que evaluaran y corrigieran la escala de 6 factores propuesta. A partir de la revisión se sugirió la inclusión de otra escala de reconceptualización del ejercicio físico y el deporte que fue incluida en el instrumento con la aprobación del grupo de expertos. Los ítems se iniciaban con la raíz "Actualmente NO practico deporte o actividad física porque ..." y un ejemplo de ítem fue "...he perdido la supervisión de entrenadores y otros profesionales". El rango de respuesta fue desde 0 (nada cierto en mi caso) a 4 (muy cierto en mi caso).

\section{Procedimiento}

Se obtuvo aprobación por parte del comité de ética de la Universidad del primer autor. Se informó de los objetivos y la naturaleza del estudio a los participantes que tuvieron que aceptar un consentimiento informado antes de completar los cuestionarios en línea.

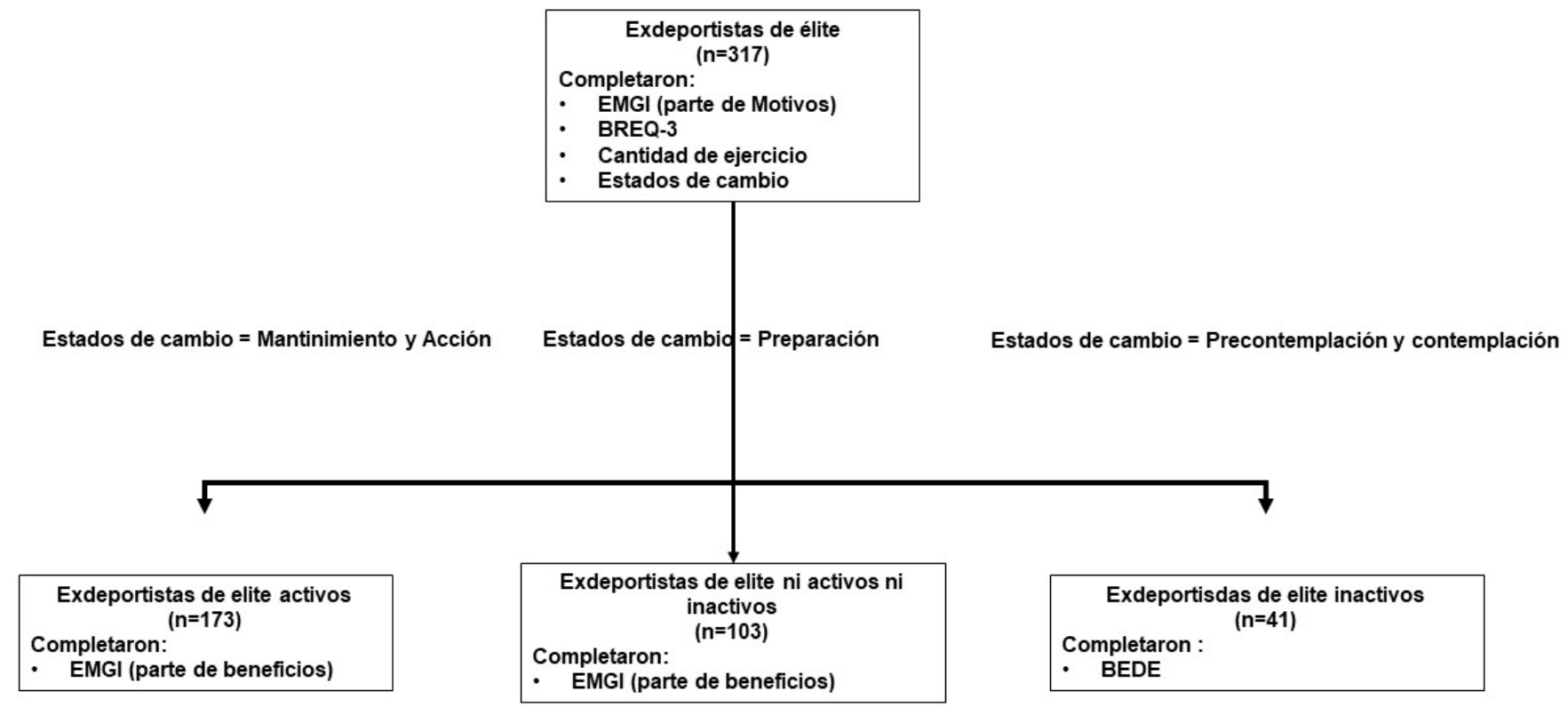

Figura 1. Diagrama de flujo de las medidas completadas por los exdeportistas de élite durante el estudio. EMGI = Inventario de Motivos y Beneficios para el Ejercicio; BREQ = Cuestionario de Regulación Conductual del Ejercicio versión 3; BEDE = Barreras para el Ejercicio físico y el Deporte recreativo percibidas por Exdeportistas de élite.

La Figura 1 resume la participación de los deportistas en el estudio. El cuestionario en línea tenía 2 partes:

1. Todos los deportistas contestaron las escalas de motivos para el ejercicio, regulación conductual, cantidad de ejercicio autoinformado y estadios de cambio. Basados en la respuesta al instrumento de estadios de cambio se clasificó a los exdeportistas en físicamente activos (i.e., aquellos en la etapa de acción y mantenimiento) o físicamente inactivos (i.e., aquellos en las etapas de precontemplación, contemplación y preparación).

2. Los exdeportistas físicamente activos contestaron el instrumento de beneficios y los físicamente inactivos contestaron el de barreras. 


\section{Torregrossa, M.; Conde, E.; Perez-Rivases, A.; Soriano, G. 1 y Ramis, Y.}

Realizamos una prueba piloto de las medidas para comprobar los instrumentos y el procedimiento. A raíz de la prueba piloto aquellos en el estado de preparación que se habían asignado originalmente a la categoría de exdeportistas físicamente inactivos se transfirieron al grupo de deportistas activos y contestaron el cuestionario de beneficios. A pesar de que de acuerdo con la teoría dichos deportistas no pueden aún experimentar los beneficios del ejercicio y el deporte de recreativo, contestaron que se sentían extraños contestando el cuestionario de barreras porque no reflejaba correctamente su situación vigente.

La versión final del cuestionario en línea incluía: (a) 152 ítems para los exdeportistas físicamente activos que empleaban en completarlo 18 minutos en promedio, y (b) 129 ítems para los exdeportistas físicamente activos que empleaban en completarlo 17 minutos en promedio.

\section{Análisis estadístico}

Se calcularon estadísticos descriptivos y diferencias de medias utilizando SPSS (SPSS, 2009). Para el cálculo de medias basado en los estados de cambio dividimos la muestra entre exdeportistas activos físicamente (i.e., estados de mantenimiento y acción; $\mathrm{n}=173 ; 54.6 \%)$ y exdeportistas físicamente inactivos (i.e., estados de precontemplación y contemplación; $\mathrm{n}=41 ; 12.9 \%$ ). Los exdeportistas en el estado de preparación $(\mathrm{n}=103 ; 32.5 \%)$ no se incluyeron en el análisis a pesar de que contestaron el cuestionario de beneficios de acuerdo con la teoría no habían estado activos durante suficiente tiempo para percibir los beneficios del ejercicio físico y el deporte recreativos.

Para el cálculo del modelo predictivo estructural utilizamos MPLUS (Muthén y Muthén,
2012). Se escogió como variable de resultado la actividad física vigorosa autoinformada dado que, de acuerdo con Haskell et al. (2007), es un indicador de mantenimiento y promoción de salud para adultos en general, y de acuerdo con Witkowski y Spangenburg (2008), esta es una necesidad especialmente relevante en el caso de los exdeportistas de élite.

\section{RESULTADOS}

\section{Resultados descriptivos}

La Tabla 1 muestra los estadísticos descriptivos (rango, media y desviación estándar) para los motivos, las regulaciones conductuales, los beneficios, las barreras y la cantidad de ejercicio físico. Adicionalmente, reportamos el coeficiente de consistencia interna alfa de Cronbach para cada subescala. Los motivos principales de los exdeportistas para hacer ejercicio físico y deporte recreativo son la buena salud y la diversión seguidos de la revitalización, la evitación de la mala salud y la gestión del estrés. Los motivos menos importantes para hacer ejercicio y deporte recreativo fueron las presiones de salud y el reconocimiento social. Los coeficientes alfa para los motivos presentan un rango entre .77 (diversión) y .93 (competición) siendo todas aceptables y similares a las reportadas previamente en el estudio original (Markland y Ingledew, 1997). En referencia a las regulaciones conductuales los exdeportistas reportaron altos niveles de regulación intrínseca, identificada e integrada. Niveles moderados de regulación introyectada $(M=2.18)$ y niveles bajos de regulación externa y amotivación. Las alfas para las regulaciones conductuales fueron todas más altas de .70 excepto para la escala de amotivación (.66) con un valor inferior debido probablemente a la falta de variabilidad en las respuestas.

Tabla 1. Estadísticos descriptivos (rango, media, desviación estándar y alfa de cronbach).

\begin{tabular}{lcccc}
\hline & Rango & $M$ & $D S$ & $\alpha$ \\
\hline Motivos (n=317) & $0-4$ & & & \\
Buena salud & & 3,51 & 0,66 &, 79 \\
Diversión & & 3,41 & 0,68 &, 77 \\
Revitalización & & 3,30 & 0,69 &, 62 \\
Evitación de mala salud & & 3,12 & 0,93 &, 88 \\
Gestión del estrés & 3,10 & 0,90 &, 88 \\
Agilidad & 2,77 & 1,13 &, 90 \\
Fuerza y resistencia & & 2,72 & 1,05 &, 87
\end{tabular}

Cuadernos de Psicología del Deporte, 19, 2 (mayo) 


\section{Deporte después del deporte}

\begin{tabular}{|c|c|c|c|c|}
\hline & Rango & $M$ & $D S$ & $\alpha$ \\
\hline Control del peso & & 2,69 & 1,26 & 91 \\
\hline Apariencia & & 2,50 & 1,15 &, 86 \\
\hline Competición & & 2,40 & 1,24 & ,93 \\
\hline Afiliación & & 2,38 & 1,03 &, 87 \\
\hline Desafío & & 2,22 & 1,13 & ,89 \\
\hline Presiones de salud & & 1,55 & 1,30 &, 88 \\
\hline Reconocimiento social & & 1,48 & 1,60 & 84 \\
\hline Regulaciones conductuales $(n=312)$ & $0-4$ & & & \\
\hline Intrínseca & & 3,26 & 0,74 &, 83 \\
\hline Identificada & & 3,22 & 0,70 & ,73 \\
\hline Integrada & & 3,17 & 0,83 & ,90 \\
\hline Introyectada & & 2,18 & 1,14 &, 87 \\
\hline Externa & & 0,92 & 1,03 & ,90 \\
\hline Amotivación & & 0,61 & 0,66 & 66 \\
\hline Beneficios $(n=258)$ & $0-4$ & & & \\
\hline Diversión & & 3,47 & 0,50 & ,62 \\
\hline Revitalización & & 3,43 & 0,65 & ,79 \\
\hline Buena salud & & 3,42 & 0,60 & ,72 \\
\hline Gestión del estrés & & 3,30 & 0,78 &, 89 \\
\hline Evitación de mala salud & & 3,05 & 0,82 &, 84 \\
\hline Fuerza y resistencia & & 3,02 & 0,81 & ,83 \\
\hline Agilidad & & 2,96 & 0,84 & ,90 \\
\hline Desafío & & 2,85 & 0,96 &, 80 \\
\hline Control del peso & & 2,75 & 0,94 &, 89 \\
\hline Competición & & 2,68 & 1,13 &, 75 \\
\hline Apariencia & & 2,65 & 0,96 &, 85 \\
\hline Afiliación & & 2,63 & 1,05 &, 86 \\
\hline Reconocimiento social & & 2,20 & 1,13 &, 83 \\
\hline Presiones de salud & & 1,80 & 1,20 &, 76 \\
\hline Barreras $(n=41)$ & $0-4$ & & & \\
\hline Conciliación & & 3,25 & 1,00 &, 80 \\
\hline Forma física & & 2,96 & 1,26 &, 88 \\
\hline Acceso a recursos & & 2,74 & 1,31 & 67 \\
\hline Imagen corporal & & 1,80 & 1,23 &, 82 \\
\hline Dificultades de reconceptualización & & 1,79 & 1,00 & ,95 \\
\hline Problemas físicos & & 1,74 & 1,51 &, 80 \\
\hline Problemas psicológicos & & 1,73 & 1,24 &, 82 \\
\hline Ejercicio reportado $(\mathrm{n}=311)$ & $0-7$ & & & \\
\hline Vigoroso & & 2,08 & 1,80 & - \\
\hline Moderado & & 1,76 & 1,57 & - \\
\hline Ligero & & 3,53 & 2,44 & - \\
\hline
\end{tabular}

Nota: Las $N$ presentan un rango de 41 a 317 debido a que algunos exdeportistas contestaron el cuestionario de beneficios y otros el de barreras además de por valores faltantes ocasionales. No se presentan alfas de Cronbach del ejercicio físico reportado porque se evaluó con medidas de un solo ítem.

Los exdeportistas activos físicamente $(\mathrm{n}=$ $258 ; 81.38 \%$ ) percibieron la diversión, la revitalización y la buena salud como los principales beneficios de la práctica de ejercicio físico saludable y deporte recreativo. Los beneficios menos percibidos fueron la afiliación, el reconocimiento social y las presiones de salud. Los coeficientes alfa para las escalas de beneficios fueron todas superiores a .72 excepto la escala de diversión (.62) con un valor inferior debido probablemente a la falta de variabilidad en las respuestas.

Los exdeportistas físicamente inactivos $(\mathrm{n}=$ $41 ; 12.9 \%)$ percibieron la conciliación $(M=3.25)$ como la barrera principal para practicar ejercicio físico saludable y deporte recreativo. Su forma física actual 


\section{Torregrossa, M.; Conde, E.; Perez-Rivases, A.; Soriano, G. 1 y Ramis, Y.}

$(M=2.96)$ conjuntamente con el acceso a los recursos $(M=2.74)$ fueron las segunda y tercera barreras respectivamente. Los problemas físicos y psicológicos $(M=1.74, M=1.73)$ son las barreras que presentaron puntuaciones más bajas. Los coeficientes alfa para la escala BEDE fueron todas más altas de.80 excepto la de acceso a recursos (.67) con un valor inferior cercano a .70 .

Trescientos once exdeportistas (98.11\%) contestaron los ítems de cantidad de ejercicio físico autoinformado. Reportaron practicar actividad física vigorosa 2.08 días por semana, actividad física moderada 1.76 días por semana, y actividad física ligera 3.53 días por semana.

\section{Diferencias entre exdeportistas físicamente activos e inactivos}

La Tabla 2 muestra las diferencias de medias entre exdeportistas activos e inactivos, la prueba $t$, los valores de significación y los tamaños del efecto $(d$ de Cohen y r) para los motivos, las regulaciones conductuales y la actividad física reportada. Los exdeportistas activos reportaron valores significativamente más altos que los inactivos de buena salud y diversión como motivos de practica de actividad física saludable y deporte recreativo. De acuerdo con el criterio de Cohen (1988), el tamaño del efecto es pequeño en ambos casos $(r=.25 ; r=.20)$. Los exdeportistas inactivos reportaron niveles significativamente más altos que los activos de presiones de salud $(M=1.46, M=2.00, t=-2.32, p$ $=.021)$ como motivos para practicar actividad física saludable. El tamaño del efecto para esta diferencia es también pequeño $(r=-.19)$.

Tabla 2. Diferencias de medias entre exdeportistas de elite activos e inactivos

\begin{tabular}{|c|c|c|c|c|c|c|c|c|c|}
\hline \multicolumn{2}{|c|}{ Motivos } & \multicolumn{2}{|c|}{$\mathrm{n}=173$} & \multicolumn{2}{|c|}{$\mathrm{n}=41$} & $t$ & $p$ & $d$ Cohen & $\mathrm{r}$ \\
\hline- & Buena salud & 3,59 & 0,60 & 3,20 & 0,88 & 3,36 & ,011 & 0,52 & ,25 \\
\hline - & Diversión & 3,53 & 0,53 & 3,22 & 0,94 & 2,75 &, 050 & 0,41 & ,20 \\
\hline - & Revitalización & 3,37 & 0,62 & 3,30 & 0,83 & 0,68 &, 531 & 0,09 &, 04 \\
\hline- & Evitación de mala salud & 3,10 & 0,86 & 3,22 & 1,05 & $-0,74$ & ,461 & $-0,12$ &,- 06 \\
\hline- & Gestión del estrés & 3,10 & 0,88 & 3,07 & 1,02 & 0,21 &, 831 & 0,03 &, 01 \\
\hline - & Agilidad & 2,72 & 1,15 & 2,85 & 1,85 & $-0,63$ &, 531 & $-0,08$ &,- 04 \\
\hline - & Fuerza y resistencia & 2,75 & 1,05 & 2,68 & 1,15 & 0,39 & ,696 & 0,06 & 03 \\
\hline- & Control del peso & 2,59 & 1,26 & 2,73 & 1,41 & $-0,64$ & 519 & $-0,10$ &,- 05 \\
\hline - & Apariencia & 2,49 & 1,14 & 2,47 & 1,29 & 0,06 & ,950 & 0,01 &, 01 \\
\hline- & Competición & 2,43 & 1,22 & 2,40 & 1,25 & 0,16 & ,872 & 0,02 & 01 \\
\hline- & Afiliación & 2,50 & 1,01 & 2,41 & 1,23 & 0,46 & ,643 & 0,07 & 03 \\
\hline - & Desafío & 2,20 & 1,17 & 2,44 & 1,17 & $-1,17$ &, 241 & $-0,20$ &,- 10 \\
\hline- & Presiones de salud & 1,46 & 1,32 & 2,00 & 1,41 & $-2,32$ & ,021 & $-0,39$ &,- 19 \\
\hline - & Reconocimiento social & 1,48 & 1,14 & 1,71 & 1,44 & $-0,96$ &, 340 & $-0,17$ &,- 08 \\
\hline \multicolumn{10}{|c|}{ Motivación } \\
\hline- & Intrínseca & 3,42 & 0,57 & 2,74 & 1,13 & 3,75 & ,001 & 0,76 & ,35 \\
\hline - & Identificada & 3,34 & 0,56 & 2,83 & 1,06 & 2,96 &, 005 & 0,60 & ,29 \\
\hline- & Integrada & 3,35 & 0,69 & 2,57 & 1,06 & 4,50 & $<, 001$ & 0,87 & , 40 \\
\hline - & Introyectada & 2,20 & 1,13 & 1,82 & 1,25 & 1,88 & ,061 & 0,32 & , 16 \\
\hline- & Externa &, 79 & 0,97 & 1,06 & 1,04 & $-1,62$ & , 106 & $-0,27$ &,- 13 \\
\hline - & Amotivación &, 56 & 0,69 & 0,85 & 0,66 & $-2,46$ & ,015 & $-0,43$ &,- 21 \\
\hline \multicolumn{10}{|c|}{ Ejercicio reportado } \\
\hline- & Vigoroso & 2,66 & 1,84 & 0,51 & 1,12 & 9,61 & $<, 001$ & 1,41 & ,58 \\
\hline- & Moderado & 1,99 & 1,63 & 0,44 & 0,78 & 8,93 & $<, 001$ & 1,21 &, 52 \\
\hline - & Ligero & 3,90 & 2,42 & 2,00 & 2,11 & 5,03 & $<, 001$ & 0,84 & ,39 \\
\hline
\end{tabular}

Nota $:$ act $=$ activos; ina $=$ inactivos 


\section{Deporte después del deporte}

Comparando ambos grupos, los exdeportistas activos reportaron niveles más altos de motivación intrínseca $(M=3.42, M=2.74, t=3.75, p=.001) \mathrm{y} d e$ regulaciones identificada e integrada $(M=3.34, M=$ $2.83, t=2.96, p=.005 ; M=3.35, M=2.57, t=4.50$, $p<.001)$. Los tres tamaños del efecto son pequeños y presentan un rango de $r=.40$ para la regulación integrada a $r=.29$ para la regulación identificada. Además, los deportistas físicamente inactivos reportaron niveles significativamente más altos de amotivación $(M=0.56, M=0.85, t=-2.46, p=.015)$, y el tamaño del efecto fue pequeño $(r=-.21)$.

Los exdeportistas físicamente activos reportaron niveles de actividad física significativamente más altos que los inactivos, confirmando la clasificación hecha a partir de los estados de cambio. Las diferencias fueron significativas en las tres categorías, vigorosa $(M=$ 2.66, $M=.51, t=9.61, p<.001)$, moderada $(M=1.99$, $M=.44, t=8.93, p<.001)$ y ligera $(M=3.90, M=$ $2.00, t=5.03, p<.001)$. El tamaño del efecto es medio para la actividad moderada y vigorosa $(r=.58, r=.52)$ y pequeño para a ligera $(r=.39)$.

\section{Modelo de ecuaciones estructurales}

A partir de nuestras hipótesis y las especificidades de los resultados descriptivos probamos un modelo en el que el motivo intrínseco más valorado (i.e., buena salud) predeciría positivamente la regulación intrínseca y negativamente la regulación externa. La diversión no se incluyó a causa de su baja consistencia interna. Además, se hipotetizó que algunos de los motivos extrínsecos principales (i.e., reconocimiento social, competición, presiones de salud y control del peso) predecirían negativamente la regulación intrínseca y positivamente la regulación externa. En el siguiente paso la regulación intrínseca predeciría positivamente el ejercicio físico vigoroso reportado mientras que la regulación externa lo predeciría negativamente. A pesar de que algunas de las predicciones no fueron en la dirección esperada, este modelo mostró un ajuste aceptable a los datos: $\chi^{2}(\mathrm{df})=875.063$ (303), $p$ $<.001$, RMSEA $($ CI 90) $=.077(.071-.083)$, CFI $=.968, \mathrm{TLI}=.963$. Considerando las influencias significativas, los resultados apoyaron parcialmente el modelo hipotetizado (ver Figura 2). En primer lugar, el motivo de buena salud predijo positivamente la regulación intrínseca y negativamente la externa. En contra de lo esperado el motivo de Competición también predijo positivamente la regulación intrínseca y el de Reconocimiento Social predijo positivamente ambas regulaciones. Tal como se había hipotetizado las Presiones de Salud y el Control del Peso predijeron negativamente la regulación intrínseca y positivamente la externa. Respecto a la predicción de la actividad física vigorosa, la motivación intrínseca la predijo en positivo y la externa la predijo negativamente. El análisis de los valores de $\mathrm{R}^{2}$ muestra que el modelo explica un porcentaje de variancia aceptable de variables endógenas: $R^{2}$ Regulación intrínseca $=.401, \mathrm{R}^{2}$ Regulación externa $=.631, \mathrm{R}^{2}$ Actividad fisica vigorosa $=.234$. 


\section{Torregrossa, M.; Conde, E.; Perez-Rivases, A.; Soriano, G. 1 y Ramis, Y.}

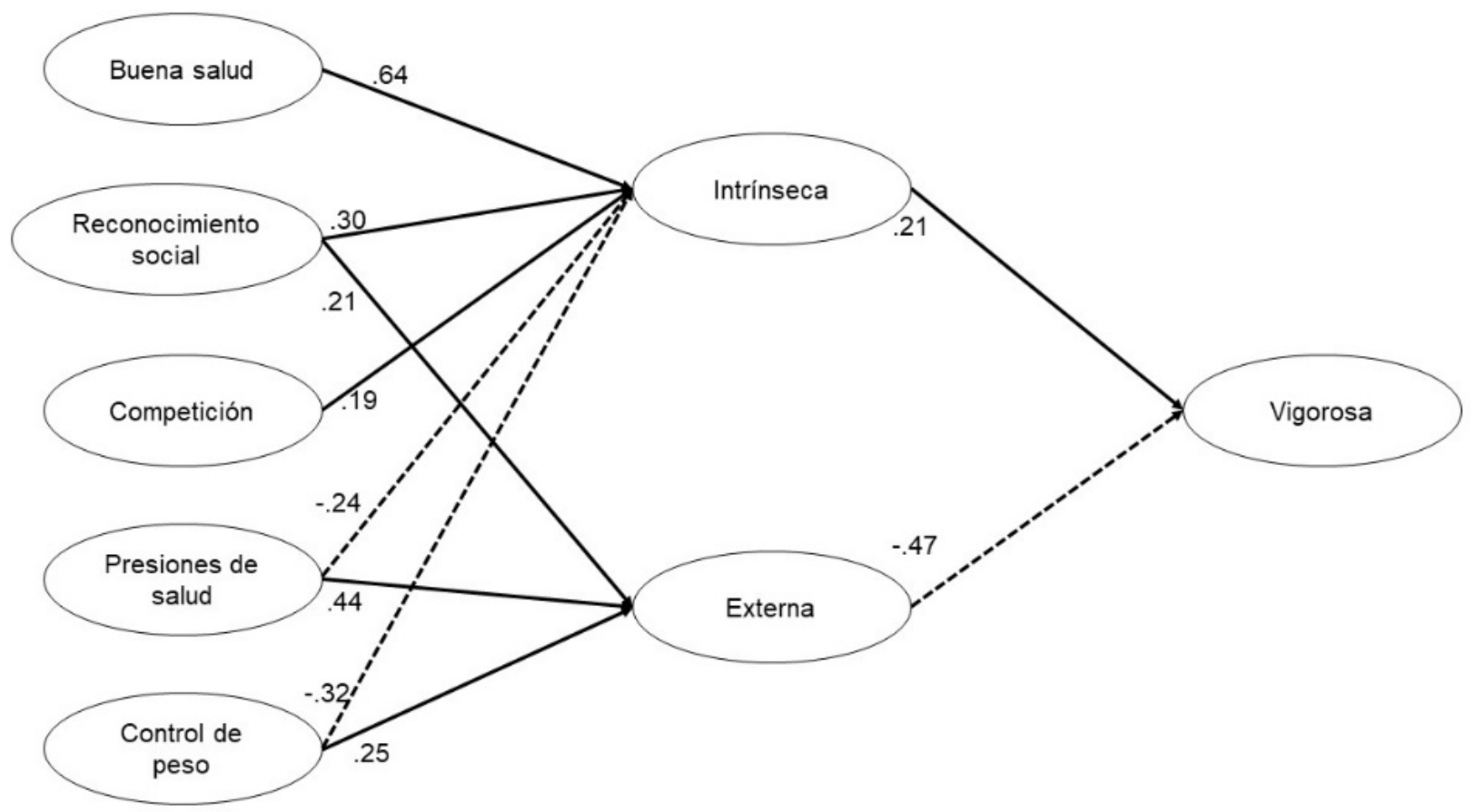

Figura 2. Modelo de motivos y regulaciones conductuales para la actividad física vigorosa en exdeportistas de élite. Nota. Las líneas punteadas indican influencia negativa.

\section{DISCUSIÓN}

El propósito de este estudio fue describir aspectos motivacionales y beneficios percibidos de exdeportistas físicamente activos y las barreras percibidas por exdeportistas físicamente inactivos para practicar actividad física saludable y deporte recreativo. También exploramos qué aspectos motivacionales predicen la práctica de actividad física vigorosa en esta población. En línea con estudios previos (e.g., Markland y Ingledew, 2007) nuestros resultados muestran que la buena salud y la diversión son motivos necesarios para la participación en ejercicio físico saludable y deporte recreativo también en exdeportistas de élite. Sin embargo, a diferencia de los estudios previos con otras poblaciones (Ingledew et al., 2014) el desafío no se considera un motivo tan importante para los exdeportistas de élite. Probablemente, esto se deba a su relación pasada con la competición de élite y la disminución de sensación de desafío que implican respecto a sus formas actuales de deporte recreativo y ejercicio físico saludable. Los motivos menos importantes son el reconocimiento social y las presiones de salud. Habiendo ya experimentado reconocimiento social por su ejecución deportiva durante su carrera parecería que no lo esperan por la actividad física saludable y el deporte recreativo en la actualidad. El hecho de no percibir las presiones de salud como un motivo para hacer ejercicio físico saludable y deporte recreativo se debe considerar como un problema potencial teniendo en cuenta el riesgo mayor de enfermedades crónicas relacionadas con su actividad física pasada que puede afectar a los exdeportistas de élite (Witkowski y Spangenburg, 2008).

En consonancia con la mayoría de estudios sobre actividad física (Teixeira et al., 2012) los niveles altos de regulaciones conductuales intrínseca e identificada y los niveles bajos de regulación externa $\mathrm{y}$ amotivación son un indicador positivo para niveles globales de cantidad y calidad de actividad física saludable practicada por los exdeportistas de élite. Los beneficios principales de practicar deporte recreativo y actividad física saludable percibidas por los exdeportistas de élite son la diversión, la revitalización y la buena salud. Siguiendo a Ingledew et al., (2014) podríamos esperar que el efecto aditivo de motivos y beneficios altos generaran regulaciones más autónomas y un efecto positive en la intención de 


\section{Deporte después del deporte}

ejercitarse, la cantidad de practica y la satisfacción con el ejercicio.

En relación a las barreras percibidas por los exdeportistas de élite el conflicto con otras actividades, su actual forma física y el hecho de haber perdido acceso a instalaciones y recursos son las que obtienen puntuaciones mayores, resultados similares a los de otras poblaciones (King et al., 2000; Niñerola et al., 2006; Zunft et al., 1999). Sin embargo, esta similitud tiene un matiz diferencial en cuanto a lo que historia personal en relación al ejercicio de nuestra población se refiere. Por un lado, en nuestra muestra la forma física actual es más baja que la que desarrollaron durante su carrera deportiva pero es aún mayor que la de la población general, por otro lado, el acceso a recursos e instalaciones en el caso de exdeportistas de élite se refiere a instalaciones, entrenadores, preparadores y médicos de alto rendimiento a los que dejaron de terner acceso al terminar sus carreras deportivas (Perez-Rivases, Miró, Pallarès y Torregrossa, 2016). El hecho de que los problemas físicos sean la barrera menos percibida es un resultado prometedor en términos de enfatizar los aspectos motivacionales y cognitivos (e.g., creencias) como clave para promocionar la actividad física saludable $y$ el deporte recreativo entre los exdeportistas de élite (Jordana, Torregrossa, Regüela, Vega y Ramis, 2018).

Los niveles de actividad reportados por nuestra muestra de exdeportistas indican un grupo remarcablemente activo en términos de actividad física vigorosa si los comparamos con el $60 \%$ de adultos en Europa que no están implicados en ningún deporte o actividad física (Eurobarometer, 2010). Nuestros resultados para los exdeportistas clasificados como físicamente activos en base a los estados de cambio (54.6\%) son muy similares a los que Bäckmand et al., (2010) encontraron con exdeportistas de élite finlandeses. Estos autores clasificaron el 54\% de los exdeportistas en el grupo físicamente más activo. En la misma línea, la proporción de exdeportistas de élite inactivos de nuestro estudio $(12.9 \%)$ es muy similar a la encontrada en el estudio de Bäckmand et al. (13.1\%). Estos resultados sugieren que en el caso de los exdeportistas de élite el problema de la inactividad física, aunque afecta a un porcentaje pequeño de esta población puede ser especialmente delicado. De acuerdo con nuestra muestra de estudio una proporción importante de los exdeportistas de élite tienen hábitos saludables en términos de actividad física y deporte recreativo autoinformados que les protegerían de los potenciales problemas que esta población tiene vinculados a los hábitos de vida sedentarios (Witkowski y Spangenburg, 2008). Sin embargo, entre un 10 y un $15 \%$ de los exdeportistas de élite tienen hábitos poco sanos en términos de ejercicio físico saludable y deporte recreativo y para ellos existe la necesidad de generar actividad física y deporte recreativo para prevenir problemas de salud (Jordana et al. 2018).

De forma consistente con estudios previos con otras poblaciones y en otros contextos, la regulación intrínseca incrementa la participación y la regulación extrínseca la reduce (Ingledew y Markland, 2008), los exdeportistas activos físicamente muestran un patrón diferenciado de medias reportando niveles significativamente más altos de motivos intrínsecos (i.e., buena salud y diversión), regulación interna (i.e., intrínseca, identificada e integrada), y actividad física autoinformada (i.e., vigorosa, moderada y ligera) que los exdeportistas físicamente inactivos. Por contra, los exdeportistas físicamente inactivos muestran niveles significativamente más altos de motivos extrínsecos (i.e., presiones de salud) y amotivación. Este perfil menos saludable en el caso de los exdeportistas de élite inactivos sugiere que a pesar de ser una proporción relativamente pequeña de la población se requiere asesoramiento específico para promover actividad física saludable y deporte recreacional enfatizando aspectos motivacionales intrínsecos.

En relación al segundo objetivo, los resultados del modelo de ecuaciones estructurales, apoyan la hipótesis de que formas de motivación más autónomas favorecen la actividad física saludable y el deporte recreativo de exdeportistas de élite como Teixeira et al., (2012) han sugerido para otras poblaciones en su revisión sistemática (e.g., adultos sanos, practicantes de ejercicio). En el caso de los exdeportistas, la buena salud, el reconocimiento social y la competición tienen un efecto positivo en la regulación interna que a su vez influye positivamente en la actividad física vigorosa. Además, las presiones de salud y el control del peso tienen un efecto negativo en la motivación intrínseca. Los motivos de control del peso, presiones de salud y reconocimiento social además, a través de su efecto positivo en la regulación externa, tienen un efecto negativo en la actividad física vigorosa. Ingledew y Markland (2008) en su estudio con oficinistas encontraron resultaos similares en relación con la buena salud, las presiones de salud y el control del 


\section{Torregrossa, M.; Conde, E.; Perez-Rivases, A.; Soriano, G. 1 y Ramis, Y.}

peso. Sin embargo, un resultado único de nuestro estudio en comparación con los anteriores es que los motivos de competición en caso de los exdeportistas de élite contribuyen a la regulación intrínseca y los de reconocimiento social tanto a las regulación intrínseca como la externa. Estos resultados sugieren por un lado, un alto grado de internalización de la competición por parte de los exdeportistas de élite, sugiriendo que estos disfrutan de manera inherente de aquellas actividades físicas o deportivas en las que la competición está presente, efecto totalmente opuesto a lo que ocurre en la población general; por otro lado, el reconocimiento social predice positivamente tanto la motivación intrínseca (i.e., crece el disfrute intrínseco a la propia actividad cuando existe reconocimiento de otros) como la externa (i.e., sentirse obligado por otros a participar en la actividad). En resumen, aquellos que diseñen programas con el objetivo de promover actividad física saludable y deporte recreativo para exdeportistas de élite deberían enfatizar la promoción de formas autónomas de motivación tomando en consideración la consistencia con la investigación previa en el papel de la buena salud, las presiones de salud y el control del peso. Sin embargo, también deberían reconocer el papel importante que, específicamente en esta población, tienen la competición y el reconocimiento social como predictores específicos de la motivación y la actividad física.

\section{Fortalezas y limitaciones}

Según consta a los autores el presente artículo, puede considerarse el primero que presenta aspectos motivacionales relacionados con la actividad física saludable y el deporte recreativo practicado por exdeportistas de élite y viene a complementar los ya realizados con exdeportistas universitarios (Reifsteck et al., 2016). Este estudio presenta datos sobre la proporción de exdeportistas de élite inactivos que, tomados conjuntamente con los de estudios pasados (Bäckmand et al., 2010), podrían interpretarse como un indicador de los niveles de deporte recreativo y actividad física saludable en exdeportistas de élite. Sin embargo, se necesitan más estudios para asegurar la representatividad de la muestra respecto a la población general de exdeportistas de élite. Además, la medida de la actividad física en términos de autopercepción es también una limitación y debería complementarse con medidas objetivas de la misma en estudios futuros.
La naturaleza transversal del estudio es también una limitación, aunque proporciona una primera imagen fija de los niveles de actividad física saludable y deporte recreativo reportada por los exdeportistas de élite. En el futuro, estudios longitudinales deberían proporcionar datos más claros sobre la evolución de la relación entre los exdeportistas de élite y el ejercicio físico saludable.

\section{CONCLUSIONES}

Nuestro estudio indica que los exdeportistas de élite pueden considerarse en general un grupo activo en términos de actividad física saludable y deporte recreativo. Sin embargo, para promover ejercicio físico saludable y deporte recreativo en el grupo inactivo, los promotores de programas deberían aportar un relato positivo enfatizando los elementos de buena salud, el reconocimiento social y la competición que predicen positivamente la regulación intrínseca y la actividad física vigorosa en esta población.

\section{APLICACIONES PRÁCTICAS}

Este trabajo tiene implicaciones prácticas relevantes. Tal como ha mostrado la literatura científica previa y corrobora nuestra investigación es muy importante que los exdeportistas de elite continúen realizando actividad física saludable después de la retirada. Concretamente, para potenciar dicha participación los profesionales aplicados deben ayudar a los exdeportistas de elite a reconceptualizar el deporte y la actividad física. Además, es importante enfatizar los motivos de buena salud, reconocimiento social y competición que llevan a los exdeportistas a formas intrínsecas de motivación y a mayor cantidad de ejercicio físico saludable y vigoroso.

\section{Agradecimientos}

Esta investigación fue apoyada, en parte, por el Proyecto DEP2014-52481-C3-1-r del Ministerio de Economía y Competitividad.

\section{REFERENCIAS}

1. Abel, M. G., y Carreiro, B. (2011). Preparing for the Big Game: Transitioning from Competitive Athletics to a Healthy Lifestyle. Strength and Conditioning Journal, 33(2), 58-63. http://doi.org/10.1519/SSC.0b013e31820bc314 


\section{Deporte después del deporte}

2. Bäckmand, H., Kujala, U., Sarna, S., y Kaprio, J. (2010). Former Athletes' Health-Related Lifestyle Behaviours and Self-Rated Health in Late Adulthood. International Journal of Sports Medicine, $\quad 31(10), \quad 751-758$. http://doi.org/10.1055/s-0030-1255109

3. Capdevila, LL. (1999). Actividad Física y Estilo de vida saludable. Terrassa: Cardellach, S.A.

4. Carek, P. J., Laibstain, S. E., y Carek, S. M. (2011). Exercise for the treatment of depression and anxiety. International Journal of Psychiatry in Medicine, 41(1), 15-28. http://doi.org/10.2190/PM.41.1.C

5. Cohen, J. (1988). Statistical power analysis for the behavioural scienes (2nd ed.). Hillsdale, NJ: Lawrence Earlbaum Associates.

6. De Bourdeaudhuij, I., y Sallis, J. (2002). Relative contribution of psychosocial variables to the explanation of physical activity in three population-based adult samples. Preventive Medicine, 34(2), 279-88. http://doi.org/10.1006/pmed.2001.0979

7. Deci, E. L., y Ryan, R. M. (2000). The "What" and "Why" of Goal Pursuits : Human Needs and the Self- Determination of Behavior. Psychological Inquiry, 11(4), 227-268. http://doi.org/10.1207/S15327965PLI1104

8. Dimoula, F., Torregrosa, M., Psychountaki, M., y Gonzalez, M. D. (2013). Retiring from elite sports in Greece and Spain. The Spanish Journal of Psychology, 16(e38), 1-11. http://doi.org/10.1017/sjp.2013.18

9. Eurobarometer. (2010). Sport and Physical Activity.

10. González-Cutre, D., Sicilia, A., y Fernández, A. (2010). Hacia una mayor comprensión de la motivación en el ejercicio físico: medición de la regulación integrada en el contexto español. Psicothema, 22(4), 841-847.

11. Haskell, W. L., Lee, I. M., Pate, R. R., Powell, K. E., Blair, S. N., Franklin, B. A., Macera, C., Heath, G., Thompson, P., y Bauman, A. (2007). Physical activity and public health: Updated recommendation for adults from the American College of Sports Medicine and the American Heart Association. Medicine and Science in Sports and Exercise, 39(8), 1423-1434. http://doi.org/10.1249/mss.0b013e3180616b27

12. Ingledew, D. K., y Markland, D. (2008). The role of motives in exercise participation. Psychology y Health, 23(7), 807-28. http://doi.org/10.1080/08870440701405704

13. Ingledew, D. K., Markland, D., y Ferguson, E. (2009). Three Levels of Exercise Motivation. Applied Psychology: Health and Well-Being, 1(3), 336-355. http://doi.org/10.1111/j.1758$\underline{0854.2009 .01015 . x}$

14. Ingledew, D. K., Markland, D., y Medley, A. R. (1998). Exercise motives and stages of change. Journal of Health Psychology, 3(4), 477-89. http://doi.org/10.1177/135910539800300403

15. Ingledew, D. K., Markland, D., y Strömmer, S. T. (2014). Elucidating the Roles of Motives and Gains in Exercise Participation. Sport, Exercise, and Performance Psychology, 3(2), 116-131. http://doi.org/10.1037/spy0000004

16. Jordana, A., Torregrosa, M., Ramis, Y., \& Latinjak, A. (2017). Retirada del deporte de élite: Una revisión sistemática de estudios cualitativos. Revista de Psicología Del Deporte, 26(4), 68-74.

17. Jordana, A., Torregrossa, M., Regüela, S., Vega, J., y Ramis, Y. (2018, diciembre). Re-engaging inactive former elite athletes in healthy sport and physical activity. Poster presentado en la Division of Sport \& Exercise Psychology Annual Conference, Belfast, Reino Unido.

18. King, A. C., Castro, C., Wilcox, S., Eyler, A. A., Sallis, J. F., y Brownson, R. C. (2000). Personal and environmental factors associated with physical inactivity among different racial-ethnic groups of U.S. middle-aged and older-aged women. Health Psychology, 19(4), 354-364. http://doi.org/10.1037/0278-6133.19.4.354

19. López de Subijana, C., Barriopedro, M., y Conde, E. (2015). Supporting dual career in Spain: Elite athletes' barriers to study. Psychology of Sport and Exercise, 21, 57-64. https://doi.org/10.1016/j.psychsport.2015.04.012

20. Marcos Pardo, P. J., Orquín Castrillón, F. J., Belando Pedreño, N., \& Moreno-Murcia, J. A. (2014). Motivación autodeterminada en adultos mayores practicantes de ejercicio físico. 


\section{Torregrossa, M.; Conde, E.; Perez-Rivases, A.; Soriano, G. 1 y Ramis, Y.}

Cuadernos de Psicología del Deporte, 14(3), 149-

156.

https://doi.org/10.4321/S157884232014000300016

21. Markland, D., y Ingledew, D. K. (1997). The measurement of exercise motives: Factorial validity and invariance across gender of a revised Exercise Motivations Inventory. British Journal of Health Psychology, 2(4), 361-376. http://doi.org/10.1111/j.20448287.1997.tb00549.x

22. Markland, D., y Ingledew, D. K. (2007). Exercise participation motives: A self-determination theory perspective. En Hagger M.S. y N. L. D. Chatzisarantis (Eds.), Self-Determination Theory in Exercise and Sport (pp. 23-34). Champaign, Ill.: Human Kinetics.

23. Markland, D., y Tobin, V. (2004). A modification of the Behavioural Regulation in Exercise Questionnaire to include an assessment of amotivation. Journal of Sport \& Exercise Psychology, 26, 191-196. http://doi.org/10.1123/jsep.26.2.191

24. Mihovilovic, M. (1968). The status of former sportsmen. International Review of Sport Psychology, 3, 79-93.

25. Moreno-Murcia, J. A., Huéscar, E., y Cervelló, E. (2012). Prediction of Adolescents doing Physical Activity after Completing Secondary Education. The Spanish Journal of Psychology, 15(1), 90100.

http://doi.org/10.5209/rev_SJOP.2012.v15.n1.37 $\underline{288}$

26. Muthén, L. K., y Muthén, B. O. (2012). MPlus User's Guide. Seventh Edition. Los Angeles: CA: Muthén \& Muthén.

27. Navel, A., y de Tychey, C. (2011). Les déterminants de la qualité du processus de reconversion sportive: revue de littérature. Bulletin de Psychologie, 513(3), 275-286. http://doi.org/10.3917/bupsy.513.0275

28. Niñerola, J., Capdevila, L., y Pintanel, M. (2006). Barreras percibidas y actividad física: el autoinforme de barreras para el ejercicio físico. Revista de Psicologia Del Deporte, 15, 53-69.

29. Park, S., Lavallee, D., y Tod, D. (2013). Athletes' career transition out of sport: A systematic review.
International Review of Sport and Exercise Psychology, 6 (1),22-53. doi:10.1080/ 1750984X.2012.687053

30. Perez-Rivases, A., Miró, S., Pallarès, S., y Torregrosa, M. (2016, Agosto). Creating a tailored physical activity programme through a focus group technique. Poster presentado en la '5th International Conference for Qualitative Research in Sport and Exercise', Chichester, UK.

31. Prochaska, J., y DiClemente, C. (1984). The transtheoretical approach: Crossing traditional boundaries of therapy. Homewood, Illinois: Dow Jones-Irwin.

32. Reichert, F. F., Barros, A. J. D., Domingues, M. R., y Hallal, P. C. (2007). The Role of Perceived Personal Barriers to Engagement in Leisure-Time Physical Activity. American Journal of Public Health, 97(3), 515-519. http://doi.org/10.2105/AJPH.2005.070144

33. Reifsteck, E. J., y Gill, D. L. (2011). Athletic identity and physical activity levels among former college athletes. Journal of Sport and Exercise Psychology, 33(Supl), 180-181.

34. Reifsteck, E. J., Gill, D. L., y Labban, J. D. (2016). "Athletes" and "Exercisers": Understanding Identity, Motivation, and Physical Activity Participation in Former College Athletes. Sport, Exercise, and Performance Psychology, 5(1), 2538. http://doi.org/10.1037/spy0000046

35. Samperio, J., Jiménez-Castuera, R., Lobato, S., Leyton, M., \& Claver, F. (2016). Variables motivacionales predictoras de las barreras para la práctica de ejercicio físico en adolescentes. Cuadernos de Psicología Del Deporte, 16(2), 6575.

36. Scarapicchia, T. M. F., Amireault, S., Faulkner, G., y Sabiston, C. M. (2016). Social support and physical activity participation among healthy adults: a systematic review of prospective studies. International Review of Sport and Exercise Psychology, 10, 50-83. http://doi.org/10.1080/1750984X.2016.1183222

37. Sørensen, M., y Gill, D. L. (2008). Perceived barriers to physical activity across Norwegian adult age groups, gender and stages of change. Scandinavian Journal of Medicine \& Science in 


\section{Deporte después del deporte}

Sports, $\quad 18(5), \quad 651-63$.

http://doi.org/10.1111/j.1600-0838.2007.00686.x

38. Stambulova, N., Alfermann, D., Statler, T., y Côté, J. (2009). ISSP Position stand: Career development and transitions of athletes. International Journal of Sport and Exercise Psychology, 7(4), 395-412. http://doi.org/10.1080/1612197X.2009.9671916

39. Stambulova, N. B., y Wylleman, P. (2015). Dual career development and transitions. Psychology of Sport and Exercise, 21, 1-3. http://doi.org/10.1016/j.psychsport.2015.05.003

40. Stephan, Y., Torregrosa, M., y Sanchez, X. (2007). The body matters: Psychophysical impact of retiring from elite sport. Psychology of Sport and Exercise, 8(1), 73-83. http://doi.org/10.1016/j.psychsport.2006.01.006

41. Teixeira, P. J., Carraça, E. V, Markland, D., Silva, M. N., y Ryan, R. M. (2012). Exercise, physical activity, and self-determination theory: a systematic review. The International Journal of Behavioral Nutrition and Physical Activity, 9(1), 78. http://doi.org/10.1186/1479-5868-9-78

42. Torregrosa, M., Ramis, Y., Pallarès, S., Azócar, F., y Selva, C. (2015). Olympic athletes back to retirement: A qualitative longitudinal study. Psychology of Sport and Exercise, 21, 50-56. http://doi.org/10.1016/j.psychsport.2015.03.003

43. Wilson, P. M., Rodgers, W. M., Loitz, C. C., y Scime, G. (2006). It's who I am...really!" The importance of integrated regulation in exercise contexts. Journal of Biobehavioral Research, 11, 79-104.

44. Witkowski, S., y Spangenburg, E. (2008). Reduced physical activity and the retired athlete: a dangerous combination? British Journal of Sports Medicine, 42, 952-953. http://doi.org/10.1136/bjsm2008.054353

45. Zunft H.J., Friebe, D., Seppelt, B., Widhalm, K., Remaunt de Winter, A. M. R., Vaz de Almeida, M. D., Kearney, J. M., y Gibney, M. (1999). Perceived benefits and barriers to physical activity in a nationally representative sample in the European Union. Public Health Nutrition, 2, 153160. http://doi.org/10.1017/S1368980099000208 